\title{
Improved EEG Source Localization with Bayesian Uncertainty Modelling of Unknown Skull Conductivity
}

\author{
Ville Rimpiläinen ${ }^{\mathrm{a}, *}$, Alexandra Koulouri ${ }^{\mathrm{b}, \mathrm{c}}$, Felix Lucka ${ }^{\mathrm{d}, \mathrm{e}}$, Jari P. Kaipio ${ }^{\mathrm{f}, \mathrm{g}}$, \\ Carsten H. Wolters ${ }^{\mathrm{h}}$ \\ ${ }^{a}$ Department of Physics, University of Bath, Claverton Down, Bath BA2 7AY, The United \\ Kingdom \\ ${ }^{b}$ Laboratory of Mathematics, Tampere University of Technology, P. O. Box 692, 33101 \\ Tampere, Finland \\ ${ }^{c}$ Department of Physics, Aristotle University of Thessaloniki, Thessaloniki 541 24, Greece \\ ${ }^{d}$ Computational Imaging, Centrum Wiskunde ES Informatica, Science Park 123, 1098 XG \\ Amsterdam, The Netherlands \\ ${ }^{e}$ Centre for Medical Image Computing, University College London, Gower Street, London \\ WC1E 6BT, The United Kingdom \\ ${ }^{f}$ Department of Mathematics, University of Auckland, Private bag 92019, Auckland 1142, \\ New Zealand \\ ${ }^{g}$ Department of Applied Physics, University of Eastern Finland, FI-90211 Kuopio, Finland \\ ${ }^{h}$ Institute for Biomagnetism and Biosignalanalysis, University of Münster, Malmedyweg 15, \\ D-48149 Münster, Germany
}

\begin{abstract}
Electroencephalography (EEG) source imaging is an ill-posed inverse problem that requires accurate conductivity modelling of the head tissues, especially the skull. Unfortunately, the conductivity values are difficult to determine in vivo. In this paper, we show that the exact knowledge of the skull conductivity 5 is not always necessary when the Bayesian approximation error (BAE) approach is exploited. In BAE, we first postulate a probability distribution for the skull conductivity that describes our (lack of) knowledge on its value, and model the effects of this uncertainty on EEG recordings with the help of an additive error term in the observation model. Before the Bayesian inference, the likelihood is marginalized over this error term. Thus, in the inversion we estimate only our primary unknown, the source distribution. We quantified the improvements in the source localization when the proposed Bayesian modelling was used in the presence of different skull conductivity errors and levels of measurement noise. Based on the results, BAE was able to improve the source localization accuracy, particularly when the unknown (true) skull conductivity was much lower than
\end{abstract}

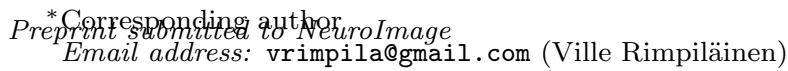

October 23, 2018 
the expected standard conductivity value. The source locations that gained the highest improvements were shallow and originally exhibited the largest localization errors. In our case study, the benefits of BAE became negligible when the signal-to-noise ratio dropped to $20 \mathrm{~dB}$.

Keywords: Electroencephalography, uncertainty modelling, Bayesian inverse problem, skull conductivity, source localization

\section{Introduction}

Electroencephalography (EEG) is a routinely used brain imaging modality to study cognitive states of the brain and to diagnose, for example, epilepsy and brain dysfunction. The EEG data is recorded by measuring electric potentials on the scalp that are induced by electric current sources in the brain [1]. EEG

25 is relatively easy to use, low-cost, it has high temporal resolution, and the apparatus is small compared to magnetoencephalography (MEG) or magnetic resonance imaging (MRI) equipment.

The mapping that translates the neuronal current sources into EEG measurements is called the forward model. The inversion of this mapping, i.e., source reconstruction from EEG data, is an ill-posed inverse problem, and stable estimates cannot be computed from noisy measurements without either regularization or by employing prior models. Additionally, the solution depends strongly on such forward model parameters as the geometry of the head [2, 3, 4, 5, 6, 7, 8, and the electric conductivies of the head tissues $9,10,11,12,13,14$. Especially the accurate conductivity modelling of the skull has been shown to be essential for accurate source reconstructions $[9,12,5,13,15,16$.

Usually, the geometry can be extracted sufficiently by using such auxiliary imaging tools as MRI and in some clinical cases computed tomography (CT) [17. However, there are only few techniques to determine or calibrate tissue 40 conductivities in vivo. These techniques usually utilize either well defined somatosensory evoked potentials / fields (SEP/SEF) in combination with EEG [18, 19, EEG/MEG [20, 21, 22, 23, 24] or electrical impedance tomography 
(EIT) [25, 26, 27]. Unfortunately, these measurements may not always be available or cannot be conducted. Moreover, even if SEP/SEF data were available for the calibration, the resulting skull conductivity value might not be optimal for sources in another brain region [19].

In this paper, we propose to use a Bayesian approximation error (BAE) approach [28, 29] to consider the unknown skull conductivity. In BAE, the observation model is formulated using a standard forward model accompanied with an additive approximation error term that encompasses the effects of the skull conductivity uncertainty. Before the Bayesian inference, this approximation error term is marginalized from the likelihood using a Gaussian approximation. Thus, the marginalized likelihood includes statistical knowledge of both the approximation error and measurement noise, and in the inversion only the unknown source configuration is solved.

The approximation error statistics are pre-computed from Monte Carlo simulations. In practice, we postulate a probability distribution for the skull conductivity and generate a set of lead field models based on this distribution. By using these sample models, we estimate the statistics of the approximation error term, i.e., the statistics of the discrepancies in the EEG recordings with respect to a standard model with a fixed skull conductivity. The posterior results from the marginalized likelihood and prior distribution of choice. The maximum-aposteriori (MAP) estimate can be used for source visualization. Previously, the BAE approach has been successfully used, for example, in EIT [30, 31, 32] and optical tomography 33, 34, 35]. In EEG source imaging, the BAE approach has been shown to alleviate localization errors arising from the unknown head geometry by using simulated 2-dimensional finite element (FE) models [36].

In the current study, we present a comprehensive analysis of the performance of the BAE approach in improving the source localization when the skull conductivity is unknown. We quantify the effects of the unknown skull conductivity by evaluating the corresponding approximation errors in the EEG recordings and the source localization errors in the reconstructions. Subsequently, we quantify the improvements in the source localization when the proposed Bayesian mod- 
elling is used in the presence of different skull conductivity errors and levels of measurement noise. This work is a simulation study that is carried out by using 3-dimensional FE-based head models.

\section{Theory}

\subsection{Bayesian framework with linear forward model}

The computational domain is denoted with $\Omega$ and its electric conductivity with $\sigma(x)$ where $x \in \Omega$. For numerical implementations, the domain is discretized and the observation model is written as

$$
v=A(\sigma) d+e
$$

where $v \in \mathbb{R}^{m}$ are the measurements, $m$ is the number of measurements, $A(\sigma) \in$ $\mathbb{R}^{m \times 3 n}$ is the lead field matrix that depends on electric conductivity $\sigma, d \in$ $\mathbb{R}^{3 n}$ is the distributed dipole source configuration and $e \sim \mathcal{N}\left(e_{*}, \Gamma_{e}\right)$ is the ${ }_{85}$ measurement noise. The noise covariance matrix is modelled as $\Gamma_{e}=\widetilde{\Gamma}_{e}+$ $\gamma I$ where the small diagonal matrix $\gamma I$ ensures that the level of noise is not underestimated.

In the Bayesian framework, the inverse solution is formally the posterior density of the Bayes formula [28, 37, 38. Here, we consider the posterior that

90 is the probability density of different source configurations given that the EEG measurements are known

$$
\pi(d \mid v) \propto \pi(v \mid d) \pi(d)
$$

where $\pi(v \mid d)$ is the likelihood and $\pi(d)$ the prior.

For the observation model, Equation (1), the likelihood model can be written as

$$
\pi(v \mid d) \propto \exp \left(-\frac{1}{2}\left(v-A d-e_{*}\right)^{\mathrm{T}} \Gamma_{e}^{-1}\left(v-A d-e_{*}\right)\right) .
$$

95 Note that the model $A(\sigma)=A$ assumes that the accurate values of electric conductivities of the patient are known which in practice, without additional effort, is almost never the case. 


\subsection{Bayesian uncertainty modelling}

In BAE, we re-write the observation model with the help of an approximate

lead field, $A\left(\sigma_{0}\right)=A_{0}$, in which we employ standard fixed values for the electric conductivity, $\sigma_{0}$. We can re-write

$$
v=A_{0} d+\varepsilon+e,
$$

where $\varepsilon=A d-A_{0} d$ is the approximation error, $\varepsilon \in \mathbb{R}^{m}$, induced by the use of the approximate model. The approximation error is a random variable (vector) whose distribution is determined by the joint (prior) distribution $\pi(\sigma, d)$.

We further approximate that $\varepsilon \sim \mathcal{N}\left(\varepsilon_{*}, \Gamma_{\varepsilon}\right), e$ and $d$ are uncorrelated, i.e., $\varepsilon$ is considered as another random additive error term. This specific BAE model is sometimes referred to as enhanced error model [28, 39, 29]. Even though, in practice, $\varepsilon$ and $d$ are usually correlated, this approximation often leads to very similar inverse solutions [28, 39, 29]. In our case, since we assume that the true source activity $d$ is focal (sparse), the cross-covariances with $\varepsilon$ will be negligible.

Based on these approximations, we formulate the probability distribution of the likelihood as

$$
\pi(v \mid d) \propto \exp \left(-\frac{1}{2}\left(v-A_{0} d-\varepsilon_{*}-e_{*}\right)^{\mathrm{T}}\left(\Gamma_{\varepsilon}+\Gamma_{e}\right)^{-1}\left(v-A_{0} d-\varepsilon_{*}-e_{*}\right)\right) .
$$

In the case of Gaussian additive measurement errors, $e \sim \mathcal{N}\left(e_{*}, \Gamma_{e}\right)$, we can quantitatively define the case when the approximation errors dominate measurement errors as

$$
\left\|e_{*}\right\|^{2}+\operatorname{tr} \Gamma_{e}<\left\|\varepsilon_{*}\right\|^{2}+\operatorname{tr} \Gamma_{\varepsilon}
$$

and

$$
e_{*, k}^{2}+\operatorname{var}\left(e_{k}\right)<\varepsilon_{*, k}^{2}+\operatorname{var}\left(\varepsilon_{k}\right),
$$

where $\operatorname{tr}(\cdot)$ is the trace of a matrix and $k=1, \ldots, m[29$. When these conditions

hold, BAE can be expected to improve the reconstruction results. We shall discuss this further in Section 5.3 . 


\subsection{Maximum a posteriori estimates}

In this paper, we compute maximum a posteriori (MAP) estimates of the posterior

$$
\hat{d}=\arg \max _{d} \pi(d \mid v)=\arg \max _{d}\{\pi(v \mid d) \pi(d)\}
$$

For comparison, we compute three different MAP estimates using three different likelihood models. We refer to these likelihood models as accurate, standard and BAE model, and we describe them in the following.

In the accurate likelihood model, we assume that the electrical conductivities are accurately known. Since we study here only single dipole source cases, we can write the MAP estimate based on the accurate likelihood model (3) as follows

$$
\begin{array}{r}
\hat{d}_{\mathrm{ACC}}=\min _{d}\left\{\left\|L_{e}\left(v-A d-e_{*}\right)\right\|_{2}^{2}\right\} \\
\text { s.t. } \quad\left\|d_{i}\right\| \cdot\left\|d_{j}\right\|=0 \quad \forall i \neq j,
\end{array}
$$
ductivity values $\left(\sigma_{0}\right)$ in the model we get

$$
\begin{array}{r}
\hat{d}_{\mathrm{STAN}}=\min _{d}\left\{\left\|L_{e}\left(v-A_{0} d-e_{*}\right)\right\|_{2}^{2}\right\} \\
\text { s.t. }\left\|d_{i}\right\| \cdot\left\|d_{j}\right\|=0 \quad \forall i \neq j
\end{array}
$$

From the likelihood of the BAE (5), the source configuration can be estimated as

$$
\begin{array}{r}
\hat{d}_{\mathrm{BAE}}=\min _{d}\left\{\left\|L_{\varepsilon+e}\left(v-A_{0} d-\varepsilon_{*}-e_{*}\right)\right\|_{2}^{2}\right\} \\
\text { s.t. }\left\|d_{i}\right\| \cdot\left\|d_{j}\right\|=0 \quad \forall i \neq j,
\end{array}
$$

where $\left(\Gamma_{\varepsilon}+\Gamma_{e}\right)^{-1}=L_{\varepsilon+e}^{T} L_{\varepsilon+e}$. Note that the BAE result is computed using the 140 same lead field matrix as in the standard model, Equation 10 . Moreover, it is 
worth noting that the only differences between (11) and 110 ) are the subtraction with $\varepsilon_{*}$ and that $L_{e}$ is replaced with $L_{\varepsilon+e}$. This means that the computational complexity is essentially the same when solving (11) and 10.

\section{Materials and methods}

The geometry of the head was constructed based on T1- and T2-weighted magnetic resonance images of a healthy subject measured with a $3 \mathrm{~T}$ MRscanner. The scalp, eyes, skull compacta, skull spongiosa, cerebrospinal fluid $(\mathrm{CSF})$, gray matter $(\mathrm{GM})$ and white matter $(\mathrm{WM})$ were segmented, for more details see [42, 14]. In the simulation set-up, 74 measurement electrodes were attached to the scalp, and the one closest to the right-hand-side ear was used as a reference.

The electric conductivities (in $\mathrm{S} / \mathrm{m}$ ) of the different tissues were 0.43 for the scalp [43, 9, 0.505 for the eyes 44], 1.79 for the CSF [45], 0.14 for the WM [44] and 0.33 for the GM [44. The skull conductivities of the different head models were the following:

First, we created 200 head models with skull conductivity drawn from a Gaussian distribution with mean $\sigma_{0}=0.01855 \mathrm{~S} / \mathrm{m}$ and standard deviation $\delta_{\sigma_{0}}=0.007225 \mathrm{~S} / \mathrm{m}$. This distribution was set in such a way that the two standard deviation lower $\left(\sigma_{0}-2 \delta_{\sigma_{0}}\right)$ and upper $\left(\sigma_{0}+2 \delta_{\sigma_{0}}\right)$ values were 0.0041 [46, 23, 20, 47] and 0.033 [48, respectively. We refer to these head models as sample head models.

Because the skull conductivity variations were assumed to be symmetrically around the mean value $\sigma_{0}=0.01855 \mathrm{~S} / \mathrm{m}$, we chose to use $\sigma_{0}$ in the standard head model and BAE. The skull spongiosa conductivities in all models were selected based on the spongiosa:compacta conductivity ratio 3.6:1 [49, 23.

The head geometry was discretized using a conforming tetrahedral FEM approach. For the forward simulations, the source space that covered the GM was constructed with 30,105 nodes on a regular grid with grid size $2 \mathrm{~mm}$. For the 
source locations on a regular grid with grid size $3 \mathrm{~mm}$. The forward source space was chosen in such a way that it did not contain the same coordinate points as the inverse source space. The lead field matrices used in the simulations were computed using standard piecewise linear FE basis functions with the Saint 175 Venant source modelling approach [47, 50].

\subsection{Computation of the approximation error statistics}

The approximation error statistics were created by first choosing randomly one of the sample head models, evaluating the model with randomly chosen source configuration, and finally calculating the approximation error by evaluating the standard model with the same source configuration,

$$
\varepsilon^{(j)}=A\left(\sigma^{(j)}\right) d^{(j)}-A_{0} d^{(j)}
$$

where $A\left(\sigma^{(j)}\right)$ is one of the sample models, $d^{(j)}$ random source configuration and $A_{0}$ is the standard model.

The procedure was repeated $J=200,000$ times, and these simulated error samples were used to calculate the sample mean, $\varepsilon_{*}$, and the sample covariance, $\Gamma_{\varepsilon}$, of the approximation error as

$$
\begin{aligned}
\varepsilon_{*} & =\frac{1}{J} \sum_{j=1}^{J} \varepsilon^{(j)} \\
\Gamma_{\varepsilon} & =\frac{1}{J-1} \sum_{j=1}^{J}\left(\varepsilon^{(j)}-\varepsilon_{*}\right)\left(\varepsilon^{(j)}-\varepsilon_{*}\right)^{\mathrm{T}} .
\end{aligned}
$$

\section{Results}

In this section, we shall quantify the approximation errors in the EEG data, the localization errors of the reconstructed dipoles, and subsequently, present how the Bayesian uncertainty modelling can improve the localization accuracy.

In the test cases, we used single dipole sources that located in the gray matter and oriented normal to the cortical surface. The EEG data was computed using one of the accurate lead field matrices that had skull conductivity 0.0041 
[23, 46, 20, 47, $0.0113,0.0258$ or $0.0330 \mathrm{~S} / \mathrm{m}$ [48, 23] (denoted as $\sigma_{0}-2 \delta_{\sigma_{0}}$, $\sigma_{0}-\delta_{\sigma_{0}}, \sigma_{0}+\delta_{\sigma_{0}}$ and $\left.\sigma_{0}+2 \delta_{\sigma_{0}}\right)$; however, all the reconstructions with the dipole scan algorithm, using Equations (11) and (10), were performed with the same lead field matrix that had the mean skull conductivity $\sigma_{0}=0.01855 \mathrm{~S} / \mathrm{m}$.

\subsection{Approximation errors of EEG data}

In order to quantify and visualize the approximation errors, i.e. the discrepancies between the EEG data computed from the accurate and standard model, we utilize the 2-norm of the normalized approximation error $\left(\varepsilon_{\mathrm{N}}\right)$ defined as

$$
\left\|\varepsilon_{\mathrm{N}}\right\|=\frac{\left\|A(\sigma) d-A_{0}\left(\sigma_{0}\right) d\right\|}{\left\|A_{0}\left(\sigma_{0}\right) d\right\|} .
$$

Figure 1 shows one saggital and axial plane of the human head, and every circle corresponds to one $\left\|\varepsilon_{\mathrm{N}}\right\|$ value computed from noiseless data by using a single dipole source. The accurate model on the top row was $A\left(\sigma_{0}-2 \delta_{\sigma_{0}}\right)$ and $A\left(\sigma_{0}+2 \delta_{\sigma_{0}}\right)$ in the bottom row.

\subsection{Localization errors of sources}

Here, we show how the $\left\|\varepsilon_{N}\right\|$ values result in source localization errors. Fig. 2 shows with circles the accurate locations of the sources and with arrows the locations where the source was reconstructed when the standard model (left column) and BAE (right column) were used. The colors correspond to the magnitude of the localization error. The same accurate models, $A\left(\sigma_{0}-2 \delta_{\sigma_{0}}\right)$ and $A\left(\sigma_{0}+2 \delta_{\sigma_{0}}\right)$, were used to generate the data with the sources that located on the same saggital and axial planes as in Fig. 1 .

Figure 3 shows how the localization errors depend on the depth (i.e. distance from scalp) of the source. The orange markers show the average localization error at a given source depth when the standard model was used and the black markers when the BAE was used in the reconstructions. The error bars illustrate the spread (standard deviation) of the localization error values. 


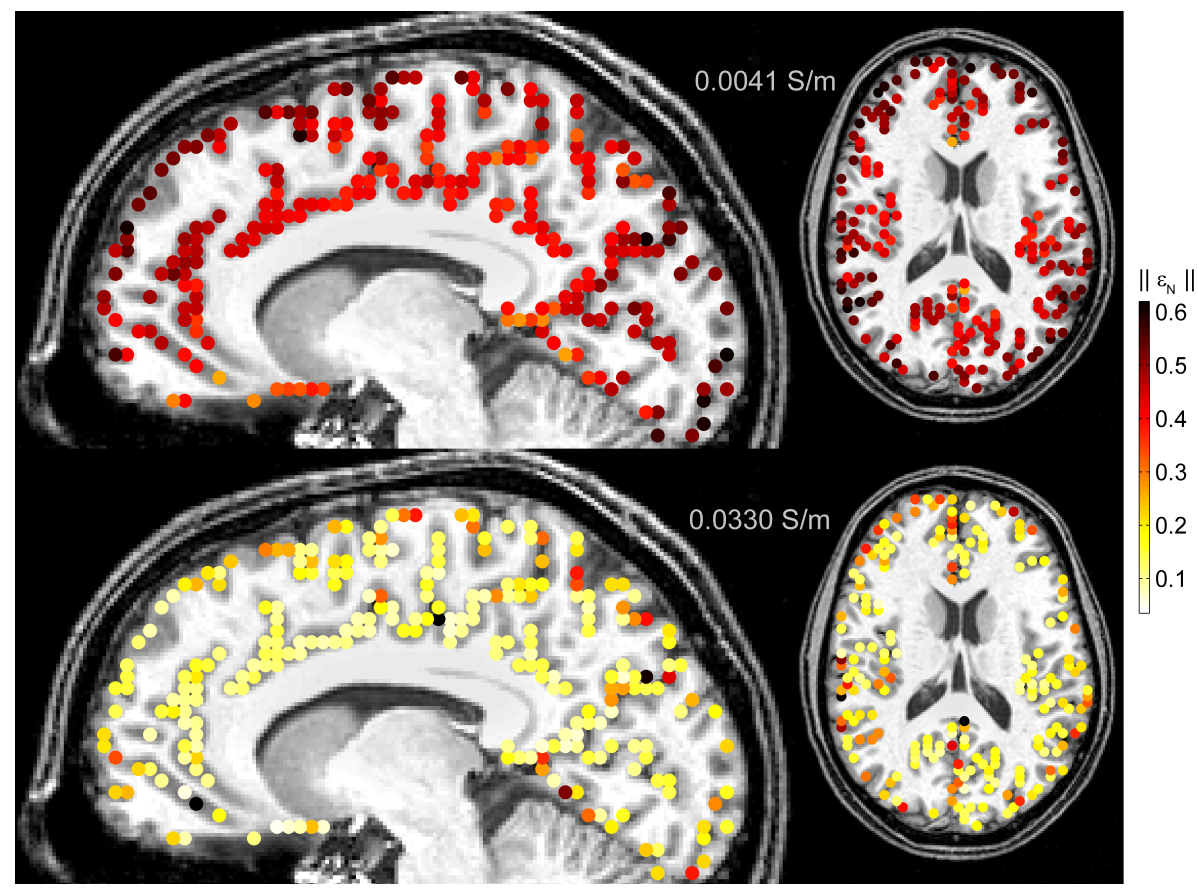

Figure 1: The 2-norm of normalized approximation error $\left(\varepsilon_{\mathrm{N}}\right)$ is shown for EEG data generated by single sources that locate in the gray matter and that are oriented normally to the cortical surface. The top row shows the $\left\|\varepsilon_{\mathrm{N}}\right\|$ values when the accurate skull conductivity in the lead field model is $0.0041 \mathrm{~S} / \mathrm{m}$ and the standard model has skull conductivity $0.01855 \mathrm{~S} / \mathrm{m}$. In the bottom row, the $\left\|\varepsilon_{\mathrm{N}}\right\|$ values are calculated assuming that $0.033 \mathrm{~S} / \mathrm{m}$ is the accurate skull conductivity. 


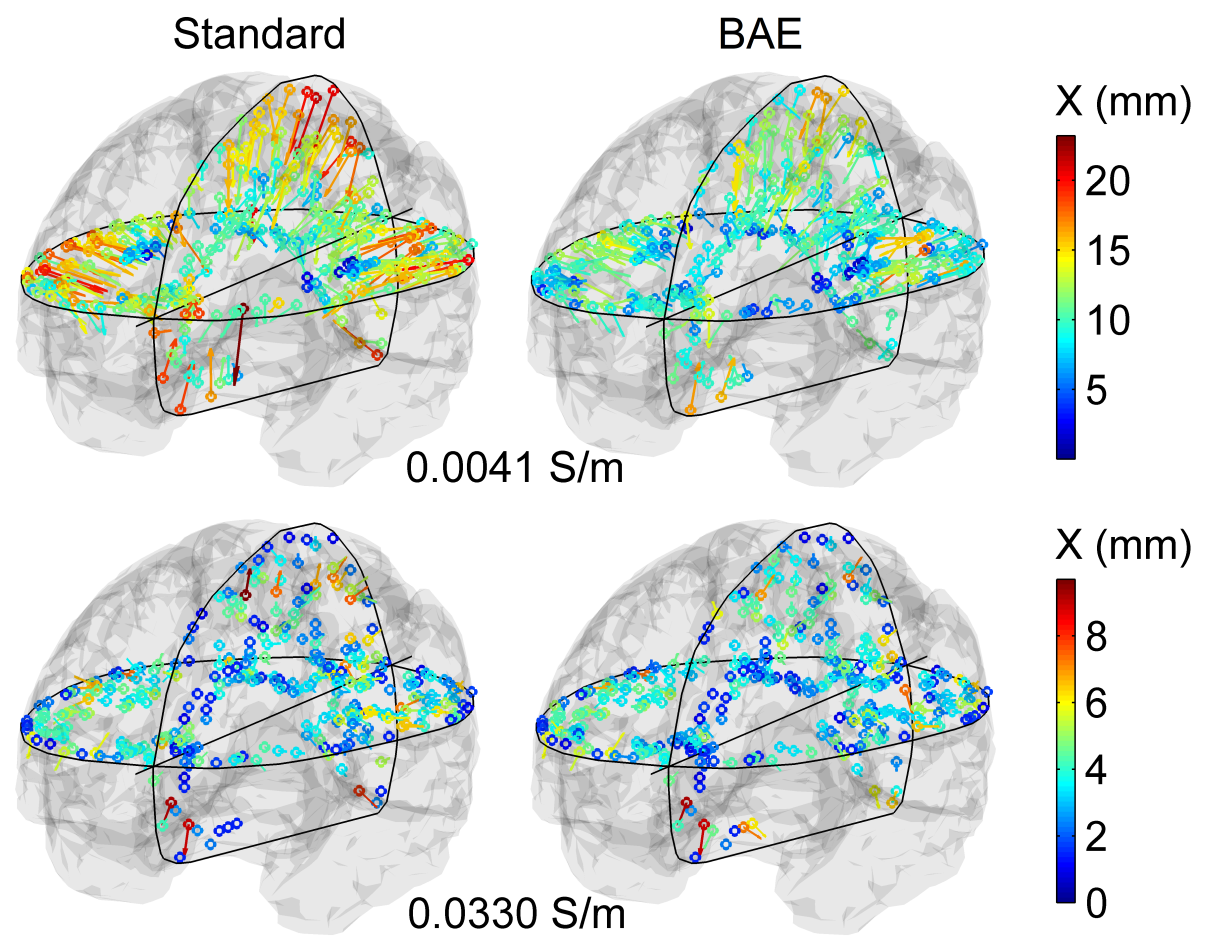

Figure 2: Top row, left: The circles show the accurate locations of the sources and the arrows show the locations of the reconstructed sources when the EEG data was generated using 0.0041 $\mathrm{S} / \mathrm{m}$ skull conductivity in the lead field model and using the standard (erroneous) $0.01855 \mathrm{~S} / \mathrm{m}$ skull conductivity in the reconstructions (left). Top row, right: These reconstructions were carried out using the BAE which takes statistically into account the expected variations in the unknown skull conductivity. Bottow row: The results are as above except that the EEG data was generated using $0.033 \mathrm{~S} / \mathrm{m}$ skull conductivity in the lead field model. 


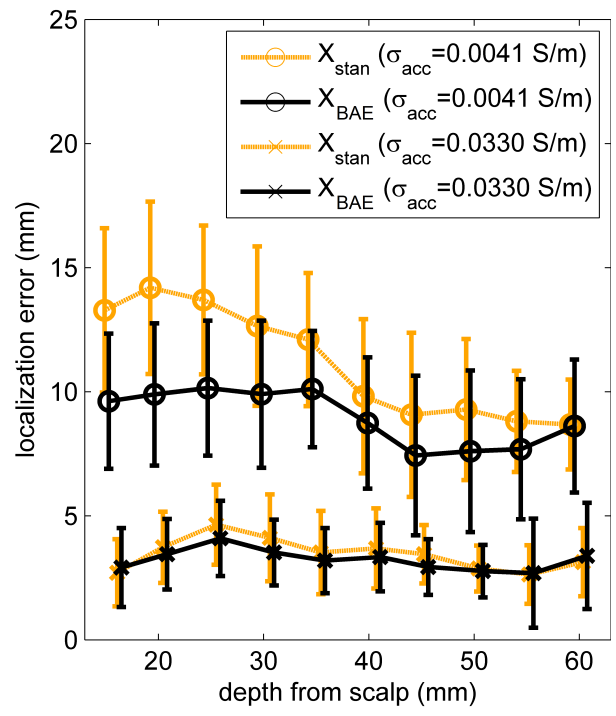

Figure 3: Localization error with respect to source depth (measured in millimeters from the scalp). The errorbars illustrate the spread (standard deviation) of the localization error values. 


\subsection{Improvement of source localization by $B A E$}

Here, we quantify the achieved improvements in the source localization when $\mathrm{BAE}$ is utilized in the inverse reconstructions. The improvement is defined as $\Delta=X_{\mathrm{STAN}}-X_{\mathrm{BAE}}$, where $X_{\mathrm{STAN}}$ and $X_{\mathrm{BAE}}$ are the localization errors evaluated from solutions of the standard model (Equation $(10 p)$ and the BAE model (Equation (11)). In these tests, we computed the EEG forward data using lead field models with skull conductivities $\sigma_{0}-2 \delta_{\sigma_{0}}, \sigma_{0}-\delta_{\sigma_{0}}, \sigma_{0}+\delta_{\sigma_{0}}$ and $\sigma_{0}+2 \delta_{\sigma_{0}}$ to showcase both small and large conductivity deviations from $\sigma_{0}$ that was used in all the reconstructions. Random white noise was added with signal-to-noise-ratios (SNR) $30 \mathrm{~dB}$ and $20 \mathrm{~dB}$. Five different noise realizations for each EEG data were drawn, five reconstructions and five distance errors were computed, and the presented $\Delta$ values are the corresponding averages.

Figure 4 presents $\Delta$ values for the $30 \mathrm{~dB}$ case with varying skull conductivity errors. $\Delta$ values are positive (red $\Delta$ signs) if BAE has improved the source localization and negative (yellow $\nabla$ signs) if BAE performs worse than the standard model. The white $\square$ signs are used if the difference of distances is less than $2 \mathrm{~mm}$.

Figure 5 shows the distributions of the $\Delta$ values in the different skull conductivity and SNR cases. Moreover, the average localization errors and improvements with their standard deviations are collected in Table 1 These values are given in millimeters between the actual and the reconstructed source for the accurate $\left(X_{\mathrm{ACC}}\right)$, standard $\left(X_{\mathrm{STAN}}\right)$ and BAE model $\left(X_{\mathrm{BAE}}\right)$. The $X_{\mathrm{ACC}}$ are given for reference. 


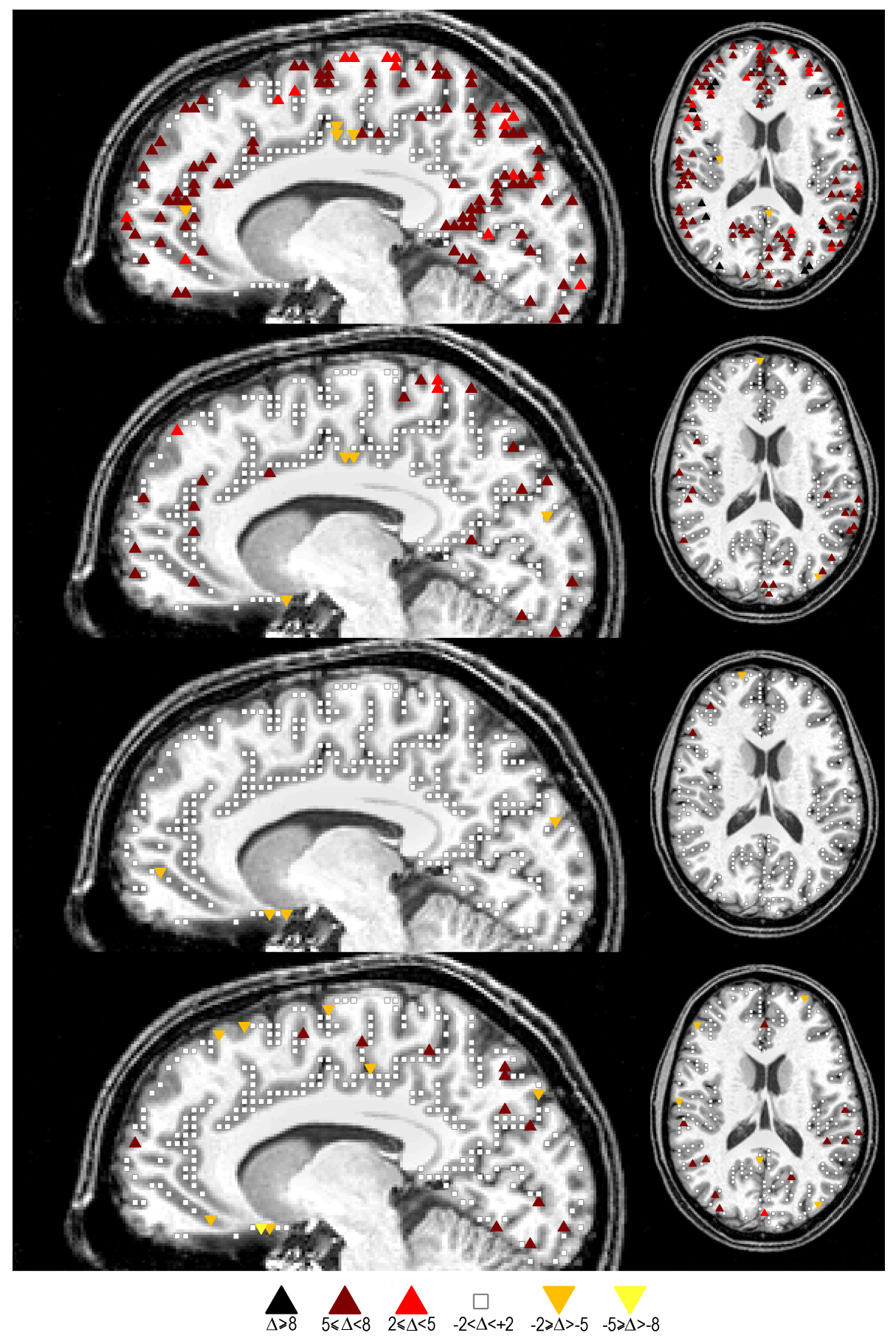

Figure 4: $\Delta$ signs show the localization improvement (in millimeters) achieved by using BAE with respect to different skull conductivity modelling errors at $\mathrm{SNR}=30 \mathrm{~dB} . \nabla$ signs are used if BAE performs worse and white $\square$ signs if the difference of the results is less than $2 \mathrm{~mm}$. In the top row, the accurate skull conductivity is $4.0041 \mathrm{~S} / \mathrm{m}$, second row $0.0113 \mathrm{~S} / \mathrm{m}$, third row $0.0258 \mathrm{~S} / \mathrm{m}$ and last row $0.033 \mathrm{~S} / \mathrm{m}$ whereas all the reconstructions are carried out by using the standard lead field matrix with fixed $0.01855 \mathrm{~S} / \mathrm{m}$ skull conductivity. 

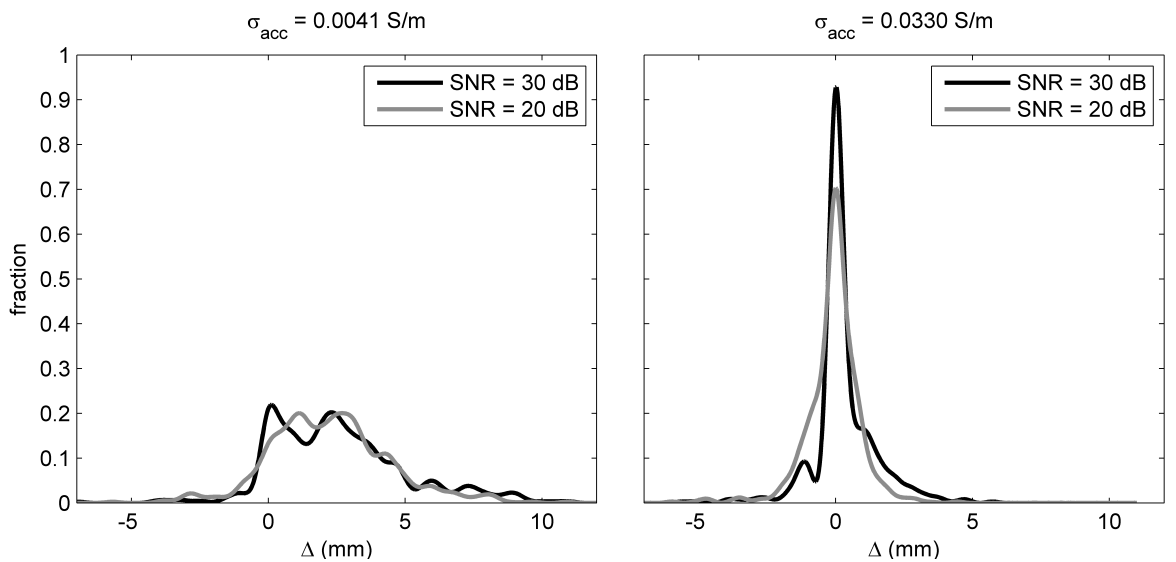

Figure 5: Left: The histograms of the improvement $\Delta$ values in the cases when the true skull conductivity was $0.0041 \mathrm{~S} / \mathrm{m}$ and SNR values $30 \mathrm{~dB}$ (black) and $20 \mathrm{~dB}$ (gray). Right: The corresponding histograms when the true skull conductivity was $0.0330 \mathrm{~S} / \mathrm{m}$. 
Table 1: To evaluate the accuracy of the different solutions, we show the averages and the \pm 1 standard deviations of the localization errors of the different models, $X_{\mathrm{ACC}}, X_{\mathrm{STAN}}$ and $X_{\mathrm{BAE}}$, respectively, and the localization improvement $\Delta$.

\begin{tabular}{cccccc}
$\begin{array}{c}\text { Testing skull } \\
\text { conductivity }(\mathrm{S} / \mathrm{m})\end{array}$ & $\begin{array}{c}\mathrm{SNR} \\
(\mathrm{dB})\end{array}$ & $\begin{array}{c}X_{\mathrm{ACC}} \\
(\mathrm{mm})\end{array}$ & $\begin{array}{c}X_{\mathrm{STAN}} \\
(\mathrm{mm})\end{array}$ & $\begin{array}{c}X_{\mathrm{BAE}} \\
(\mathrm{mm})\end{array}$ & $\begin{array}{c}\Delta \\
(\mathrm{mm})\end{array}$ \\
\hline 0.0041 & $\infty$ & $1.7 \pm 0.5$ & $12.1 \pm 3.7$ & $9.4 \pm 2.9$ & $2.8 \pm 3.1$ \\
& 30 & $1.8 \pm 0.7$ & $12.1 \pm 3.7$ & $9.8 \pm 3.1$ & $2.3 \pm 2.9$ \\
& 20 & $2.8 \pm 1.6$ & $12.2 \pm 3.9$ & $10.9 \pm 3.6$ & $1.3 \pm 2.5$ \\
\hline 0.0113 & $\infty$ & $1.7 \pm 0.5$ & $4.2 \pm 1.8$ & $3.6 \pm 1.5$ & $0.7 \pm 1.7$ \\
& 30 & $1.8 \pm 0.7$ & $4.2 \pm 1.8$ & $3.7 \pm 1.6$ & $0.5 \pm 1.6$ \\
& 20 & $2.4 \pm 1.3$ & $4.5 \pm 2.0$ & $4.2 \pm 2.0$ & $0.3 \pm 1.6$ \\
\hline 0.0258 & $\infty$ & $1.7 \pm 0.6$ & $2.6 \pm 1.1$ & $2.4 \pm 1.0$ & $0.2 \pm 1.0$ \\
& 30 & $1.8 \pm 0.7$ & $2.6 \pm 1.1$ & $2.5 \pm 1.2$ & $0.1 \pm 1.0$ \\
& 20 & $2.2 \pm 1.1$ & $3.0 \pm 1.5$ & $3.0 \pm 1.6$ & $0.0 \pm 1.2$ \\
\hline 0.0330 & $\infty$ & $1.7 \pm 0.6$ & $3.6 \pm 1.6$ & $3.3 \pm 1.5$ & $0.3 \pm 1.4$ \\
& 30 & $1.8 \pm 0.7$ & $3.6 \pm 1.6$ & $3.4 \pm 1.5$ & $0.2 \pm 1.4$ \\
& 20 & $2.2 \pm 1.1$ & $3.9 \pm 1.9$ & $3.9 \pm 1.9$ & $0.1 \pm 1.4$ \\
\hline
\end{tabular}




\section{Discussion}

\subsection{Approximation errors of EEG data}

Based on Fig. 1, we can see that $\left\|\varepsilon_{N}\right\|$ values are generally larger when the source is close to the skull (and the electrodes) than deep in the brain.

\subsection{Localization errors of sources}

As can be seen from Fig. 2, the trends are that the over-estimation of skull conductivity (the top row) moves the reconstructed sources deeper in the 

result is in agreement with literature (e.g. [51]). The over-estimation causes much larger localization errors than under-estimation which is in-line with the approximation errors that are also larger for the over-estimation case (see, Fig. 11. Therefore, it can be argued that if there is no information on the skull conductivity value rather than a high one.

When the skull conductivity is over-estimated, the sources that are close to the skull present on average higher localization errors than the ones deeper in the brain. This is because the deep sources cannot move further deeper in the brain since they are already close to the bottom boundary of the source space to begin with. Therefore, the deep sources exhibit smaller localization errors than the shallow ones.

In the under-estimation case, the localization errors are noticeably smaller. The sources that are shallow but not quite at the outer boundary have slightly the geometry of the source space: the sources close to the skull have smaller localization errors (than the slightly deeper ones) since they already locate close to the skull (top boundary of the source space) and cannot therefore move any closer.

This depth dependence is more evident in Fig. 3. These results are inline with Fig. 1 in the sense that the localization errors are larger for the source locations that also exhibit large $\left\|\varepsilon_{N}\right\|$ values. BAE can improve the source localization especially at points where the approximation and localization errors are large for the standard model. If the localization errors are small, then also the compensation by BAE is small and may be unnoticeable in the given discretization.

\subsection{Improvement of source localization by $B A E$}

When the skull conductivity is over-estimated (accurate skull conductivity is either $\sigma_{0}-2 \delta_{\sigma_{0}}$ or $\sigma_{0}-\delta_{\sigma_{0}}$ compared to $\sigma_{0}$ in the inverse model), the localiza- 

more significant.

Based on this, the consideration of the skull conductivity uncertainties by utilizing BAE is the most beneficial when the SNR of the measurements is more than $20 \mathrm{~dB}$ and it can be expected that the patient's skull conductivity may dif-

tion improvements are the largest. This is especially evident for SNR $=30 \mathrm{~dB}$ and more moderate for $20 \mathrm{~dB}$. The over-estimation, on average, induces higher approximation errors than under-estimation (as discussed in Section 5.1). The improvements gained with $\mathrm{BAE}$ are the highest, up to about $10 \mathrm{~mm}$, for the sources that locate close to the skull. This is in agreement with the locations that exhibit the highest $\left\|\varepsilon_{\mathrm{N}}\right\|$ values.

When the skull conductivity is under-estimated (accurate skull conductivity is either $\sigma_{0}+\delta_{\sigma_{0}}$ or $\sigma_{0}+2 \delta_{\sigma_{0}}$ compared to $\sigma_{0}$ in the inverse model), the improvements are smaller. On average, there are small improvements for $\mathrm{SNR}=30 \mathrm{~dB}$, but for the $20 \mathrm{~dB}$ cases BAE gives on average similar results as the standard model. The improvements gained with $\mathrm{BAE}$ are the highest for the sources that locate slightly deeper in the brain (than in the over-estimation case) even though the $\left\|\varepsilon_{N}\right\|$ values are on average higher closer to the skull. This is because the under-estimation of skull conductivity shifts the sources closer to the skull, and the sources that already locate close to the skull (top boundary of the source space) cannot shift any closer.

There are some locations for which the standard model gives accurate results, regardless of the erroneous skull conductivity. This occurs in such locations that do not have wide support (grid points) around them. For example, when the GM is highly curved there may not be additional points around the original source location where the source could be moved.

The limit where the measurement errors start to become equal to the approximation errors (see, Equation (6)) occurs when the SNR drops to $20 \mathrm{~dB}$. This deteriorates the benefits of BAE especially in cases where the $\left\|\varepsilon_{N}\right\|$ values are low, and the BAE solution, Equation (10), starts to approach the solution of the standard model, Equation (11), as the noise covariance matrix $\left(\Gamma_{e}\right)$ becomes fer (significantly) from the skull conductivity that is used in the model. There- 
fore, the proposed uncertainty modelling can be very useful, for example, when examining infants and young children whose skull conductivities are unknown since the value can range between several orders of magnitude [12, 52, 7]. With $\mathrm{BAE}$, it is not necessary to know the exact skull conductivity, and the range of possible values can be modelled as a probability distribution. To use BAE, it is advisable to set-up this probability distribution (i.e. $\sigma_{0}$ and $\delta_{\sigma_{0}}$ in our case) based on age-specific literature values (e.g. [48), then estimate the statistics of the approximation error term as described in Section 3.2 and finally add the corresponding $\varepsilon_{*}$ and $L_{\varepsilon}$ terms in the likelihood.

\subsection{Transferability of Bayesian uncertainty modelling}

The presented BAE approach is an $a$-priori procedure in the sense that it does not require any measurement or calibration data. The BAE approach is versatile since it can be accompanied with any prior model for the sources and any (linear or non-linear) source reconstruction algorithm. Furthermore, the inclusion of BAE does not increase the complexity of the source reconstruction (as pointed out in Section 2.3) and the evaluation of the BAE statistics can be performed off-line, before any measurements are carried out.

In this paper, we chose to address the well-known problem of unknown skull conductivity. In addition to this, BAE can be used to treat other unknown (or uncertain) forward model parameters, such as the geometry of the head [36. Moreover, the properties of the EEG electrodes are typically not modelled accurately and for instance their contact impedances are only poorly known [53. 54. The positions of the electrodes can carry uncertainty as they are either simply assumed to be distributed according to a standard system [55] or measured by a tracking system before the EEG recording takes place. BAE is also transferable to other imaging settings, such as MEG. In MEG source analysis, a major uncertainty is the position and orientation of the head with respect to the MEG sensor coils [56, [57].

\subsection{Methodological comparison to empirical Bayesian frameworks}


The simplicity and transferability of BAE can be highlighted when compared to empirical Bayesian frameworks [58, 59, 60, 61, 62. In an empirical Bayesian framework, the unknown forward model parameters are also treated as stochastic variables. However, unlike in BAE, in this option these forward model parameters are estimated from the measured EEG data alongside the source activity and parameters of the prior model by maximizing the Bayesian model-evidence (or a free-energy approximation thereof) [60, or marginalized by using Bayesian model averaging 63, 64]. In other words, these are a-posteriori procedures since they require and depend on the EEG measurement data which is then used to estimate both the source activity and the parameters of the forward model that is exploited for the inference. This usually results in a considerably higher computational complexity compared to plain source reconstruction or BAE. In the BAE approach, the unknown forward model parameters do not need to be evaluated, it is simply enough to address their uncertainties and encapsulate them in the approximation error term. Therefore, the computational complexity of BAE is essentially the same as in the plain source reconstruction. Furthermore, as already stated, the BAE model does not depend on any measurement data, and thus is an a-priori procedure. In conclusion, the BAE approach can be considered as a more straightforward alternative to empirical Bayesian frameworks.

\section{Conclusions and future work}

We have characterized the use of Bayesian uncertainty modelling of unknown skull conductivity in EEG source imaging with respect to different skull conductivity errors and SNRs. We have shown that modelling of the skull conductivity uncertainties can reduce the source localization errors by several millimeters, and about a centimeter in the best cases. The localization accuracy improves especially when the unknown skull conductivity is over-estimated. The underestimation of the skull conductivity causes smaller localization errors, and thus the improvements are smaller as well. The highest improvements occur for the 
sources that locate either close to the skull or slightly deeper in the brain, and this is in agreement with the locations that exhibit the most prominent localization errors. The benefits of BAE become uncertain when the SNR drops to $20 \mathrm{~dB}$. The proposed uncertainty modelling can be beneficial especially for infants or young children whose skull conductivities are unknown since the values can range between several orders of magnitude.

395

In the future studies, experimental evaluation will be carried out. In the experiments, it might be beneficial to concentrate only on a specific region of the brain that corresponds to a particular cognitive state or brain dysfunction. This anatomical restriction could further increase the localization improvements of BAE since the induced approximation errors will have more systematic patterns 400 (rather than when the whole brain is considered). In the future, we will also study the uncertainty modelling of head geometry and skin contact in combination with the conductivity uncertainties.

\section{Acknowledgement}

This work was supported in parts by the Academy of Finland post-doctoral program (project no. 316542), IKY Fellowships of excellence for postgraduate studies in Greece - Siemens program, the Engineering and Physical Sciences Research Council, UK (EP/K009745/1), the European Union's Horizon 2020 research and innovation programme H2020 ICT 2016-2017 under grant agreement No 732411 (as an initiative of the Photonics Public Private Partnership) and the Netherlands Organisation for Scientific Research (NWO 613.009.106/2383), EU project ChildBrain (Marie Curie Innovative Training Networks, grant agreement no. 641652) and by the Deutsche Forschungsgemeinschaft (DFG, project WO1425/7-1).

\section{References}

${ }_{415}$ [1] R. Brette, A. Destexhe (Eds.), Handbook of Neural Activity Measurement, Cambridge University Press, New York, 2012. 
[2] B. N. Cuffin, Effects of head shape on EEGs and MEGs, IEEE Trans. Biomed. Eng. 37 (1) (1990) 44-52. doi:10.1109/10.43614.

[3] B. N. Cuffin, Effects of local variations in skull and scalp thickness on EEG's and MEG's, IEEE Trans. Biomed. Eng. (1) (1993) 42-48.

[4] G. Huiskamp, M. Vroeijenstijn, R. van Dijk, G. Wieneke, A. C. van Huffelen, The need for correct realistic geometry in the inverse EEG problem, IEEE Trans. Biomed. Eng. 46 (11) (1999) 1281-1287. doi:10.1109/10. 797987.

[5] V. Montes-Restrepo, P. van Mierlo, G. Strobbe, S. Staelens, S. Vanderberghe, H. Hallez, Influence of skull modeling approaches on EEG source localization, Brain Topogr. 27 (2014) 95-111.

[6] S. Vallaghé, M. Clerc, A global sensitivity analysis of three- and four-layer EEG conductivity models, IEEE Trans. Biomed. Eng. 56 (2009) 988-995.

[7] H. Azizollahi, A. Aarabi, F. Wallois, Effects of uncertainty in head tissue conductivity and complexity on EEG forward modelling in neonates, Hum. Brain Mapp. 37 (2016) 3604-3622. doi:10.1002/hbm. 23263.

[8] M. Stenroos, A. Nummenmaa, Incorporating and compensating cerebrospinal fluid in surface-based forward models of magneto- and electroencephalography, PLoS ONE 11 (2016) e0159695.

[9] M. Dannhauer, B. Lanfer, C. Wolters, T. Knösche, Modeling of the human skull in eeg source analysis, Hum. Brain Mapp. 32 (2011) 1383-1399.

[10] K. Awada, D. Jackson, S. B. Baumann, J. Williams, D. Wilton, P. Fink, B. Prasky, Effect of conductivity uncertainties and modeling errors on EEG source localization using a 2-D model, IEEE Trans. Biomed. Eng. 45 (9) (1998) 1135-1145. doi:10.1109/10.709557.

[11] S. P. van den Broek, F. Reinders, M. Donderwinkel, M. J. Peters, Volume conduction effects in EEG and MEG, Electroenceph. Clin. Neurophysiol. 106 (6) (1998) 522-534. doi:10.1016/S0013-4694(97)00147-8. 
[17] P. J. Slomka, R. P. Baum, Multimodality image registration with software: state-of-the-art, Eur. J. Nucl. Med. Mol. Imaging 36 (1) (2009) 44-55. doi:10.1007/s00259-008-0941-8.

[18] S. Lew, C. Wolters, A. Anwander, S. Makeig, M. R., Improved EEG

[20] M. Fuchs, M. Wagner, H. A. Wischmann, T. Köhler, A. Theissen, R. Drenckhahn, H. Buchner, Improving source reconstructions by com- 
bining bioelectric and biomagnetic data, Electroencephalogr. Clin. Neurophysiol. 107 (2) (1998) 93-111.

[21] M. X. Huang, T. Song, D. J. H. Jr., I. Podgorny, V. Jousmaki, L. Cui, D. L. Harrington, A. M. Dale, R. R. Lee, J. Elman, E. Halgren, A novel integrated MEG and EEG analysis method for dipolar sources, NeuroImage 37 (3) (2007) 731-748.

[22] C. Wolters, S. Lew, R. S. MacLeod, M. Hämäläinen, Combined EEG/MEG source analysis using calibrated finite element models, Biomedizinische Technik/Biomedical Engineering. Rostock, Germany: Walter de Gruyter, Vol. 55 (Suppl. 1) (2010) 64-68.

[23] U. Aydin, J. Vorwerk, P. Küpper, M. Heers, H. Kugel, A. Galka, L. Hamid, J. Wellmer, C. Kellinghaus, S. Rampp, C. H. Wolters, Combining EEG and MEG for the reconstruction of epileptic activity using a calibrated realistic volume conductor model, PLoS ONE 9 (2014) e93154.

[24] U. Aydin, S. Rampp, A. Wollbrink, H. Kugel, J.-H. Cho, T. R. Knösche, C. Grova, J. Wellmer, C. H. Wolters, Zoomed MRI guided by combined EEG/MEG source analysis: A multimodal approach for optimizing presurgical epilepsy work-up and its application in a multi-focal epilepsy patient case study, Brain Topogr. 30 (2017) 417-433.

[25] S. Goncalves, J. C. de Munck, J. P. A. Verbunt, R. M. Heethaar, F. H. L. da Silva, In vivo measurement of the brain and skull resistivities using an eit-based method and the combined analysis of sef/sep data, IEEE Trans. Biomed. Eng. 50 (9) (2003) 1124-1128.

[26] S. I. Goncalves, J. C. de Munck, J. P. A. Verbunt, F. Bijma, R. M. Heethaar, F. Lopes da Silva, In vivo measurement of the brain and skull resistivities using an eit-based method and realistic models for the head, IEEE Trans. Biomed. Eng. 50 (6) (2003) 754-767. 
[27] J. Dabek, K. Kalogianni, E. Rotgans, F. C. van der Helm, G. Kwakkel, E. E. van Wegen, A. Daffertshofer, J. C. de Munck, Determination of head conductivity frequency response in vivo with optimized EIT-EEG, NeuroImage 127 (2016) 484-495.

[28] J. P. Kaipio, E. Somersalo, Statistical and Computational Inverse Problems, Applied Mathematical Series, Springer, 2004.

[29] J. Kaipio, V. Kolehmainen, Approximate marginalization over modeling errors and uncertainties in inverse problems, in: P. Damien, N. Polson, D. Stephens (Eds.), Bayesian Theory and Applications, Oxford University Press, 2013.

[30] A. Lipponen, A. Seppänen, J. P. Kaipio, Nonstationary approximation error approach to imaging of three-dimensional pipe flow: experimental evaluation,, Meas. Sci. Technol. 22 (10) (2011) 104013.

[31] A. Nissinen, L. M. Heikkinen, V. Kolehmainen, J. P. Kaipio, Compensation of errors due to discretization, domain truncation and unknown contact impedances in electrical impedance tomography, Meas. Sci. Technol. 20 (10) (2009) 105504.

[32] A. Nissinen, V. Kolehmainen, J. Kaipio, Compensation of modelling errors due to unknown domain boundary in electrical impedance tomography, IEEE Trans. Med. Imag. 30 (2) (2011) 231-242.

[33] S. R. Arridge, J. P. Kaipio, V. Kolehmainen, M. Schweiger, E. Somersalo, T. Tarvainen, M. Vauhkonen, Approximation errors and model reduction with an application in optical diffusion tomography, Inverse Problems 22 (2006) 175-195. doi:10.1088/0266-5611/22/1/010.

[34] V. Kolehmainen, M. Schweiger, I. Nissilä, T. Tarvainen, S. R. Arridge, J. P. Kaipio, Approximation errors and model reduction in three-dimensional diffuse optical tomography, J. Opt. Soc. Am. A 26 (10) (2009) 2257-2268. doi:10.1364/JOSAA.26.002257. 
[35] T. Tarvainen, V. Kolehmainen, A. Pulkkinen, M. Vauhkonen, M. Schweiger, S. R. Arridge, J. P. Kaipio, An approximation error approach for compensating for modelling errors between the radiative transfer equation and the diffusion approximation in diffuse optical tomography, Inverse Problems 26 (1) (2010) 015005. doi:10.1088/0266-5611/26/1/015005.

[36] A. Koulouri, V. Rimpiläinen, M. Brookes, J. P. Kaipio, Compensation of domain modelling errors in the inverse source problem of the Poisson equation: Application in electroencephalographic imaging, Appl. Num. Math. 106 (2016) 24-36.

[37] D. Calvetti, E. Somersalo, Introduction to Bayesian scientific computing: Ten lectures on subjective computing, Springer, 2007.

[38] A. Tarantola, Inverse problem theory and methods for model parameter estimation, Society for Industrial and Applied Mathematics, 2007.

[39] J. Kaipio, E. Somersalo, Statistical inverse problems: Discretization, model reduction and inverse crimes, J. Comput. Appl. Math. 198 (2) (2007) 493504. doi:10.1016/j.cam.2005.09.027.

[40] J. C. Mosher, P. S. Lewis, R. M. Leahy, Multiple dipole modeling and localization from spatio-temporal MEG data, IEEE. Trans. Biomed. Eng. 39 (1992) 541-557.

[41] T. R. Knösche, Solutions of the neuroelectromagnetic inverse problem, Ph.D. thesis, University of Twente, The Netherlands (1997).

[42] F. Lucka, S. Pursiainen, M. Burger, C. H. Wolters, Hierarchical Bayesian inference for the EEG inverse problem using realistic FE head models: depth localization and source separation for focal primary currents, NeuroImage 61 (4) (2012) 1364-1382.

[43] C. Ramon, P. Schimpf, J. Haueisen, M. Holmes, A. Ishimaru, Role of soft bone, CSF and gray matter in EEG simulations, Brain Topogr. 16 (2004) $245-248$. 
[44] C. Ramon, P. H. Schimpf, J. Haueisen, Influence of head models on EEG simulations and inverse source localizations, Biomed. Eng. Online 5 (10).

[45] S. B. Baumann, D. R. Wozny, S. K. Kelly, F. M. Meno, The electrical conductivity of human cerebrospinal fluid at body temperature, IEEE Trans. Biomed. Eng. 44 (1997) 220-223.

[46] S. Homma, T. Musha, Y. Nakajima, Y. Okomoto, S. Blom, R. Flink, K. E. Hagbarth, Conductivity ratios of the scalp-skull-brain head model in estimating equivalent dipole sources in human brain, Neurosci. Res. 22 (1) (1995) 51-55.

[47] H. Buchner, G. Knoll, M. Fuchs, A. Rienäcker, R. Beckmann, M. Wagner, J. Silny, J. Pesch, Inverse localization of electric dipole current sources in finite element models of the human head, Electroencephalogr. Clin. Neurophysiol. 102 (1997) 267-278.

[48] R. Hoekema, G. H. Wieneke, C. W. van Veelen, P. C. van Rijen, G. J. Huiskamp, J. Ansems, A. C. van Huffelen, Measurement of the conductivity of skull, temporarily removed during epilepsy surgery, Brain Topogr. 16 (1) (2003) 29-38.

[49] M. Akhtari, H. C. Bryant, A. N. Mamelak, E. R. Flynn, L. Heller, J. J. Shih, M. Mandelkern, A. Matlachov, D. M. Ranken, E. D. Best, M. A. DiMauro, R. R. Lee, W. W. Sutherling, Conductivities of three-layer live human skull, Brain Topogr. 14 (3) (2002) 151-167.

[50] S. Lew, C. Wolters, T. Dierkes, C. Röer, R. MacLeod, Accuracy and runtime comparison for different potential approaches and iterative solvers in finite element method based eeg source analysis, Appl. Num. Math. 59 (2009) 1970-1988.

[51] R. Pohlmeier, H. Buchner, G. Knoll, A. Rienäcker, R. Beckmann, J. Pesch, The influence of skull-conductivity misspecification on inverse source local- 
ization in realistically shaped finite element head models, Brain Topogr. 9 (1997) 157-162.

[52] M. Odabaee, A. Tokariev, S. Layeghy, M. Mesbah, P. B. Colditz, C. Ramon, S. Vanhatalo, Neonatal EEG at scalp is focal and implies high skull conductivity in realistic neonatal head models, NeuroImage 96 (2014) 7380.

[53] S. Pursiainen, F. Lucka, C. H. Wolters, Complete electrode model in EEG: relationship and differences to the point electrode model, Physics in Medicine and Biology 57 (2012) 999-1017. doi:10.1088/0031-9155/ $57 / 4 / 999$.

[54] S. Pursiainen, B. Agsten, S. Wagner, C. H. Wolters, Advanced boundary electrode modeling for tES and parallel tES/EEG, IEEE Trans. Neural Syst. Rehabil. Eng. 26 (2018) 37-44.

595 [55] R. Oostenveld, P. Praamstra, The five percent electrode system for highresolution EEG and ERP measurements, Clin. Neurophysiol. 112 (2001) $713-719$.

[56] A. Stolk, A. T. J.-M. Schoffelen, R. Oostenveld, Online and offline tools for head movement compensation in MEG, NeuroImage 68 (1) (2013) 39-48.

[57] S. Meyer, J. Bonaiuto, M. Lim, H. Rossiter, S. Waters, D. Bradbury, S. Bestmann, Flexible head-casts for high spatial precision MEG, J. Neurosci. Methods 276 (2017) 38-45.

[58] K. Friston, L. Harrison, J. Daunizeau, S. Kiebel, C. Phillips, N. TrujilloBarreto, R. Henson, G. Flandin, J. Mattout, Multiple sparse priors for the M/EEG inverse problem, NeuroImage 39 (2008) 1104-1120.

[59] R. N. Henson, J. Mattout, C. Phillips, K. J. Friston, Selecting forward models for MEG source-reconstruction using model-evidence, NeuroImage 46 (2009) 168-176. 
[60] S. T. Hansen, S. Hauberg, L. K. Hansen, Data-driven forward model inference for EEG brain imaging, NeuroImage 139 (2016) 246-258.

[61] R. Henson, D. Wakeman, V. Litvak, K. Friston, A parametric empirical bayesian framework for the EEG/MEG inverse problem: Generative models for multi-subject and multi-modal integration, Front. Hum. Neurosci. 5 (2011) 76. doi:10.3389/fnhum.2011.00076

[62] G. Strobbe, P. van Mierlo, M. D. Vos, B. Mijovi, H. Hallez, S. V. Huffel, J. D. Lpez, S. Vandenberghe, Bayesian model selection of template forward models for eeg source reconstruction, NeuroImage 93 (2014) 11 - 22. doi: https://doi.org/10.1016/j.neuroimage.2014.02.022

[63] N. J. Trujillo-Barreto, E. Aubert-Vázquez, P. A. Valdés-Sosa, Bayesian model averaging in EEG/MEG imaging, NeuroImage 21.

[64] J. D. López, W. D. Penny, J. J. Espinosa, G. R. Barnes, A general bayesian treatment for MEG source reconstruction incorporating lead field uncertainty, NeuroImage 60 (2012) 1194-1204.

\section{Appendix: Bayesian approximation error approach}

In the following, we derive the likelihood terms of the Bayesian approximation error approach.

Let's take the observation model as (4), and denote the total error as $\nu=$ $\varepsilon+e$. Because the inverse solution is the posterior density of the Bayes' formula $\pi(d \mid v) \propto \pi(v \mid d) \pi(d)$, we derive a formulation to $\pi(v \mid d)$. This is done by marginalizing $\pi(v, \nu \mid d)$ with respect to $\nu$, i.e.

$$
\pi(v \mid d)=\int_{\nu \in \mathbb{R}^{m}} \pi(v, \nu \mid d) d \nu .
$$

From the joint density $\pi(v, \nu, d)=\pi(v, \nu \mid d) \pi(d)=\pi(v \mid \nu, d) \pi(\nu \mid d) \pi(d)$, we obtain

$$
\pi(v \mid d)=\int_{\nu \in \mathbb{R}^{m}} \pi(v \mid \nu, d) \pi(\nu \mid d) \mathrm{d} \nu
$$


Now, the conditional density of $v$ given $d$ and $\nu$ is obtained by using (4) as

$$
\pi(v \mid d, \nu)=\delta\left(v-A_{0} d-\nu\right)
$$

where $\delta(\cdot)$ is the Dirac delta distribution.

Hence, from (18) and (17), we have that the likelihood $\pi(v \mid d)$ is the convolution

$$
\begin{aligned}
\pi(v \mid d) & =\int_{\nu \in \mathbb{R}^{m}} \delta\left(v-A_{0} d-\nu\right) \pi(\nu \mid d) \mathrm{d} \nu \\
& =\pi_{\nu \mid d}\left(v-A_{0} d \mid d\right)
\end{aligned}
$$

where the subscript $\nu \mid d$ is used to clarify that the probability density is that of $\nu$ given $d$. We consider that measurement noise $e \sim \mathcal{N}\left(e_{*}, \Gamma_{e}\right)$ and the approximation error term $\varepsilon_{\mid d} \sim \mathcal{N}\left(\varepsilon_{* \mid d}, \Gamma_{\varepsilon \mid d}\right)$ are mutually uncorrelated and have Gaussian distributions. The subscript $\mid d$ indicates that the approximation error $\varepsilon$ in a general case depends on $d$.

Thus, the approximate likelihood is a Gaussian distribution given by

$$
\pi_{\nu \mid d}\left(v-A_{0} d \mid d\right) \propto \exp \left(-\frac{1}{2}\left(v-A_{0} d-\nu_{* \mid d}\right)^{T} \Gamma_{\nu \mid d}^{-1}\left(v-A_{0} d-\nu_{* \mid d}\right)\right)
$$

with $\nu_{* \mid d}=\varepsilon_{* \mid d}+e_{*}$ and $\Gamma_{\nu \mid d}=\Gamma_{\varepsilon \mid d}+\Gamma_{e}$.

In this paper, we use a special case of the BAE approach in which $d$ and $\varepsilon$ are 645 treated as mutually uncorrelated variables, i.e. $\varepsilon \sim \mathcal{N}\left(\varepsilon_{*}, \Gamma_{\varepsilon}\right)$ (a.k.a. enhanced error model). Thus, we can write that $\nu_{*}=\varepsilon_{*}+e_{*}$ and $\Gamma_{\nu}=\Gamma_{\varepsilon}+\Gamma_{e}$. We conclude to the likelihood (5). For further details on the formal derivation of the likelihood, see for example [28, 29, 33]. 